\title{
ESTENOSIS URETERAL POST-TRASPLANTE RENAL: TRATAMIENTO CON PRÓTESIS METÁLICA AUTOEXPANDIBLE
}

\author{
B. POZO MENGUAL, F.J. BURGOS REVILLA*, A. LINARES QUEVEDO, G. BRIONES \\ MARDONES, J. PASCUAL SANTOS**, R. MARCEN LETOSA**, T. LLORENTE*** \\ Servicio de Urología. Hospital Ramón y Cajal. Madrid. *Dpto. Ciencias Morfológicas y Cirugía. \\ Universidad de Alcalá de Henares. Madrid. **Servicio de Nefrología. Hospital Ramón y Cajal. Madrid. \\ ***Servicio de Radiología. Hospital Ramón y Cajal. Madrid.
}

\section{Actas Urol Esp. 27 (3): 190-195, 2003 \\ RESUMEN \\ "ESTENOSIS URETERAL POST-TRASPLANTE RENAL: TRATAMIENTO CON PRÓTESIS METÁLICA AUTOEXPANDIBLE"}

OBJETIVO: Determinar la eficacia del empleo de prótesis metálicas autoexpandibles en el tratamiento de las estenosis ureterales post-trasplante renal.

MATERIAL Y MÉTODOS: Desde octubre de 1995 a abril de 2002, se han tratado 8 estenosis ureterales postTR mediante el implante de una prótesis metálica autoexpandible ( 6 hombres y 2 mujeres). El tiempo medio de seguimiento fue de 30 meses (rango 2-53 meses). En este trabajo se analizan la severidad y localización de la estenosis, el método de implante de la prótesis, la permeabilidad de la misma, los niveles de creatinina pre y postimplante y las complicaciones derivadas del mismo.

RESULTADOS: El tratamiento fue efectivo en el 100\% de los pacientes (8/8), con un descenso medio de la creatinina del 36\% (rango 13,6\%-59,6\%). Las complicaciones fueron mínimas y la estancia hospitalaria fue corta.

CONCLUSIONES: En los pacientes con: deterioro crónico del injerto con expectativa funcional corta, pacientes con alto riesgo quirúrgico y reestenosis tras reimplantación ureterovesical por estenosis ureteral previa, el empleo de una prótesis metálica autoexpandible constituye la técnica de elección dada su eficacia y baja morbilidad asociada.

PALABRAS CLAVE: Trasplante renal. Stent metálico. Estenosis ureteral.

\section{ABSTRACT \\ "URETERAL OBSTRUCTION AFTER RENAL TRANSPLANTATION: TREATMENT WITH METALLIC SELF-EXPANDABLE STENT"}

OBJECTIVE: To determinate the efficacy in the treatment of ureteral estenoses after renal transplantation with metallic self-expandable stent.

MATERIALS AND METHODS: From october of 1995 to april of 2002, 8 ureteral obstruction post renal transplantation have treated by means of implants of a metallic self-expandable stent (6 men and 2 women). The average time of pursuit was of 30 months (rank 2-53 months).

In this work the severity and location of the estenosis are analysed, the method of implant of the stent, the permeability of the same one, the levels of creatinine pre and postimplant and the complications derived from the same one.

RESULTS: The treatment was effective in the $100 \%$ of the patients $(8 / 8)$, with an average reduction of the creatinine of 36\% (rank 13,6\%-59,6\%). The complications were minimun and the hospital stay was short.

CONCLUSIONS: In the patients with: chronic deterioration of the graft with short functional expectation, patient with high surgical risk and reestenosis after ureteral reimplantation by previous ureteral estenosis, the use of a metallic self-expandable stent, constitutes technique of election given its efficacy and low associate morbidity. 
$\mathrm{L}$ as complicaciones más frecuentes que afectan a la vía urinaria post-trasplante renal (TR) son las estenosis y las fístulas ureterales, con una frecuencia que oscila entre el $2-13 \%{ }^{1}$.

La reimplantación ureteral constituye, clásicamente, la técnica de primera elección en estos procesos, presentando una morbi-mortalidad no desdeñable ${ }^{2}$. Desde el desarrollo de técnicas endourológicas en la década de los ochenta, el tratamiento de las estenosis ureterales por estos procedimientos ha supuesto una alternativa válida, con buenos resultados e índices bajos de complicaciones.

Son múltiples las opciones endourológicas que se pueden ofertar en pacientes seleccionados: colocación de catéter ureteral, dilatación con catéter-balón, implante de endoprótesis metálicas autoexpandibles, etc.

Se presenta la experiencia de nuestro grupo en el tratamiento de las estenosis ureterales post-TR mediante el empleo de endoprótesis metálicas autoexpandibles.

\section{MATERIAL Y MÉTODOS}

Desde octubre de 1995 a abril de 2002, se han tratado 8 estenosis ureterales post-TR mediante el implante de una prótesis metálica autoexpandible, correspondiendo a 6 hombres y 2 mujeres de edad media de 54 años (rango 21-69 años). La indicación del implante de la prótesis se realizó como primera elección en 4 pacientes y tras fracaso de una reimplantación ureteral por estenosis ureteral en otros 4 pacientes.

Los cuatro casos de primera indicación corresponden a: rechazo crónico con deterioro funcional avanzado ( 2 casos), enfermedad tumoral concomitante ( 1 caso) y cardiopatía severa ( 1 caso).

Los cuatro pacientes en los que la implantación del stent se realizó tras el fracaso de una segunda reimplantación ureterovesical corresponden a: 3 fístulas ureterales post-TR y 1 trombosis venosa ureteral con necrosis asociada.

El tiempo transcurrido desde el trasplante hasta el implante de la prótesis osciló entre 5 meses y 7 años (15,2 $\pm 3,6$ meses).

En los 8 casos se habían empleado técnicas extravesicales de reimplante urétero-vesical. El tiempo medio de seguimiento fue de 30 meses (rango 2-53 meses).
En este trabajo se analizan la severidad y localización de la estenosis, el método de implante de la prótesis, la permeabilidad de la misma, los niveles de creatinina pre y post-implante y las complicaciones derivadas del mismo.

En la Tabla I se resumen los datos epidemiológicos de los pacientes.

La sospecha clínica de estenosis ureteral se basó en la elevación de los productos nitrogenados.

La ecografía del injerto, siguiente paso en el diagnóstico, evidenció hidronefrosis de distinto grado en los 8 casos. Ante la confirmación ecográfica de uropatía obstructiva, se realizó pielografia anterógrada previa colocación de nefrostomía percutánea, tipo malecot $12 \mathrm{Fr}$, ecodirigida, sola o asociada a fluoroscopia convencional.

Los datos de la pielografía evaluados fueron: grado de estenosis, número, localización y longitud de la misma (Fig. 1).

En 4 pacientes la estenosis era completa y en 4 pacientes existía paso mínimo de contraste a vejiga. En todos los pacientes la estenosis era única, localizada en la unión uretero-vesical y menor de tres centímetros.

El implante del stent se realizó en un segundo tiempo a los 3-5 días de la colocación de la nefrostomía percutánea, tras la recuperación funcional del injerto.

Se utilizaron endoprótesis metálicas autoexpandibles de nitinol (Memo Therm -MOVACO) de distintos calibres y longitudes. En 6 pacientes el calibre empleado fue de $8 \mathrm{~mm}$, en 1 caso de $6 \mathrm{~mm}$ y en otro caso de $4 \mathrm{~mm}$. Las longitudes de los stent fueron: $8 \mathrm{~cm}$ ( 4 casos), $6 \mathrm{~cm}$ ( 3 casos) y $4 \mathrm{~cm}$ ( 1 caso) (Tabla II).

El procedimiento quirúrgico se indica a continuación:

En todos los pacientes se realizó profilaxis antibiótica previa a la cirugía y se realizó el tratamiento bajo sedación.

A través de la nefrostomía, se pasa guía metálica curva $0,35 \mathrm{Ch}$, con ayuda de catéter ureteral (selectip 5-7 Fr) hasta vejiga. Una vez alojada la guía en vejiga se exterioriza, empleando el cistoscopio y la pinza de cuerpo extraño, a través de la uretra.

Se realiza, a continuación, dilatación con catéter balón de alta presión (Fig. 2). Utilizando la guía como tutor y bajo control fluoroscópico se pasa de 


\section{TABLA I}

DATOS EPIDEMIOLÓGICOS DE LOS PACIENTES

\begin{tabular}{|c|c|c|c|c|c|c|}
\hline Paciente & $\begin{array}{c}\text { Edad } \\
\text { al TR }\end{array}$ & $\begin{array}{c}\text { Causa } \\
\text { IRCr }\end{array}$ & $\begin{array}{c}\text { Localización } \\
\text { injerto }\end{array}$ & $\begin{array}{c}\text { Tipo reimplante } \\
\text { U-V }\end{array}$ & $\begin{array}{c}\text { Catéter } \\
\text { ureteral }\end{array}$ & $\begin{array}{c}\text { Complicaciones } \\
\text { post-TR }\end{array}$ \\
\hline 1 & 21 & $\begin{array}{c}\text { Nefropatía } \\
\text { intersticial }\end{array}$ & FID & Extravesical & No & $\begin{array}{c}\text { Retrasplante (1991) } \\
\text { NTA } \\
\text { Fístula ureteral }\end{array}$ \\
\hline 2 & 53 & No filiada & FID & Extravesical & No & NTA* \\
RAO x HBP
\end{tabular}

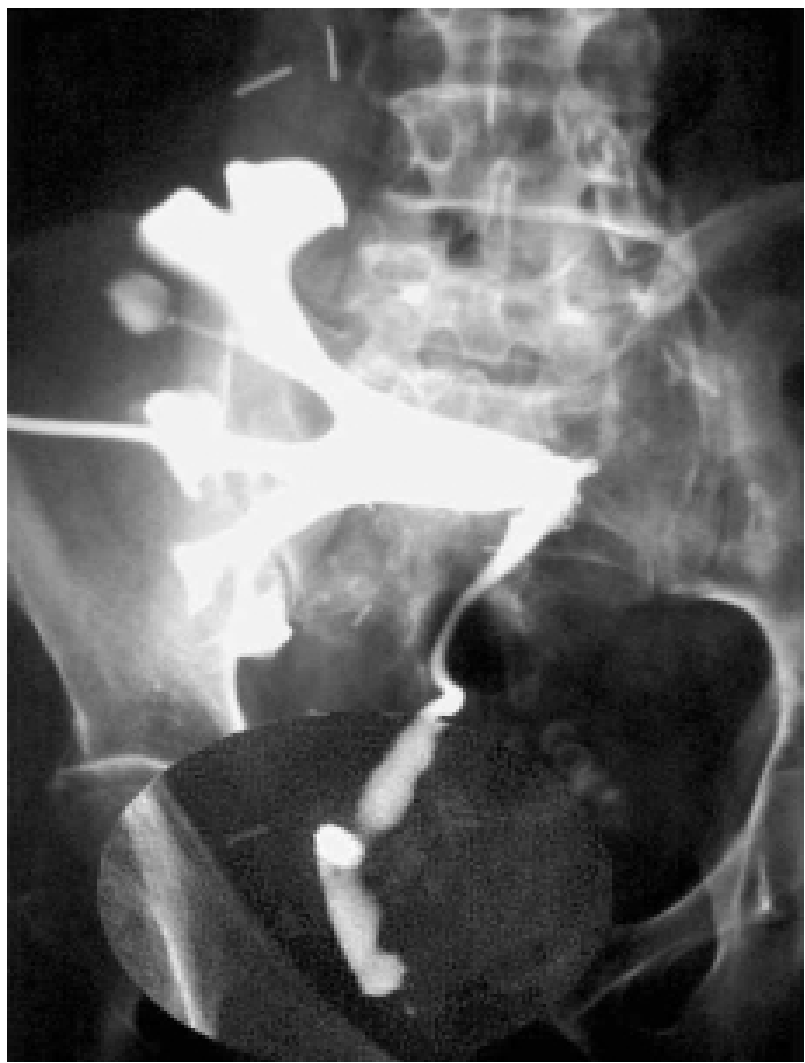

FIGURA 1. Pielografia anterógrada. Estenosis ureteral distal que impide el paso de contraste a vejiga. forma anterógrada prótesis metálica autoexpandible hasta cubrir toda la longitud de la estenosis. A continuación, se introduce cistoscopio visualizándose el extremo vesical del stent. La pinza permite fijar el extremo de la prótesis permitiendo que la misma quede enrasada con la superficie ureteral a nivel de la reimplantación sin que quede stent "flotando" endovesicalmente (Fig. 3).

Tras la colocación de la prótesis se realiza pielografía intraoperatoria para confirmar correcto implante y permeabilidad del mismo (Fig. 4).

La principal dificultad técnica radica en la tutorización de la estenosis. En nuestra serie, en uno de los casos fue necesaria la resección transuretral de la zona de ureteroneocistostomía para poder recuperar la guía metálica.

Definimos éxito de tratamiento la confirmación de permeabilidad ureteral y persistencia de la misma sin necesidad de nuevas alternativas terapéuticas, asociado a la recuperación de la función renal post-desobstructiva.

\section{RESULTADOS}

El tratamiento fue efectivo en el 100\% de los pacientes $(8 / 8)$, con un descenso medio de la cre- 
TABLA II

LONGITUD Y CALIBRE DE LOS DISTINTOS STENT EMPLEADOS

\begin{tabular}{|c|c|c|c|}
\hline \multirow{2}{*}{ Paciente } & \multicolumn{2}{|c|}{ Tipo stent } & \multirow{2}{*}{$\begin{array}{c}\text { Dilatación } \\
\text { ureteral previa }\end{array}$} \\
\cline { 2 - 4 } & $\begin{array}{c}\text { Longitud } \\
(\mathrm{cm})\end{array}$ & $\begin{array}{c}\text { Calibre } \\
(\mathrm{mm})\end{array}$ & $\mathrm{Si}$ \\
\hline 1 & 6 & 6 & $\mathrm{Si}$ \\
\hline 2 & 4 & 8 & $\mathrm{Si}$ \\
\hline 3 & 6 & 8 & $\mathrm{Si}$ \\
\hline 4 & 8 & 4 & $\mathrm{Si}$ \\
\hline 5 & 6 & 8 & $\mathrm{Si}$ \\
\hline 6 & 8 & 8 & $\mathrm{Si}$ \\
\hline 7 & 8 & 8 & $\mathrm{Si}$ \\
\hline 8 & 8 & 8 & \\
\hline
\end{tabular}

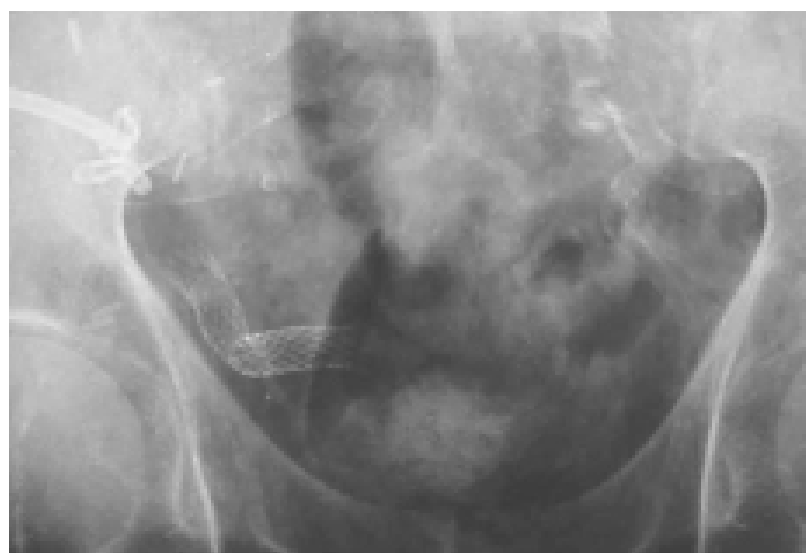

FIGURA 2. Posición adecuada del stent en la desembocadura del uréter a la vejiga sin que exista prótesis endovesical.

atinina del 36\% (rango 13,6-59,6\%). En todos los pacientes se comprobó, antes de la retirada de la nefrostomía, mediante pielografía anterógrada la permeabilidad del stent.

El paciente al que se le había colocado prótesis de menor calibre ( $4 \mathrm{~mm}$ ), presentó, en el post-operatorio inmediato, oclusión de la luz ureteral por hiperplasia urotelial, a nivel de la prótesis, resuelta con la colocación temporal de un catéter doble $\mathrm{J}$, comprobándose la permeabilidad ureteral tras la retirada del mismo a las 6 semanas.

En todos los casos la estenosis fue por causa benigna. En uno de los pacientes existieron dudas pues tenía antecedentes de uropatía obstructiva secundaria a tumor en uréter del injerto que precisó resección transuretral del mismo. A los 12 meses se detectó nueva estenosis en uréter distal

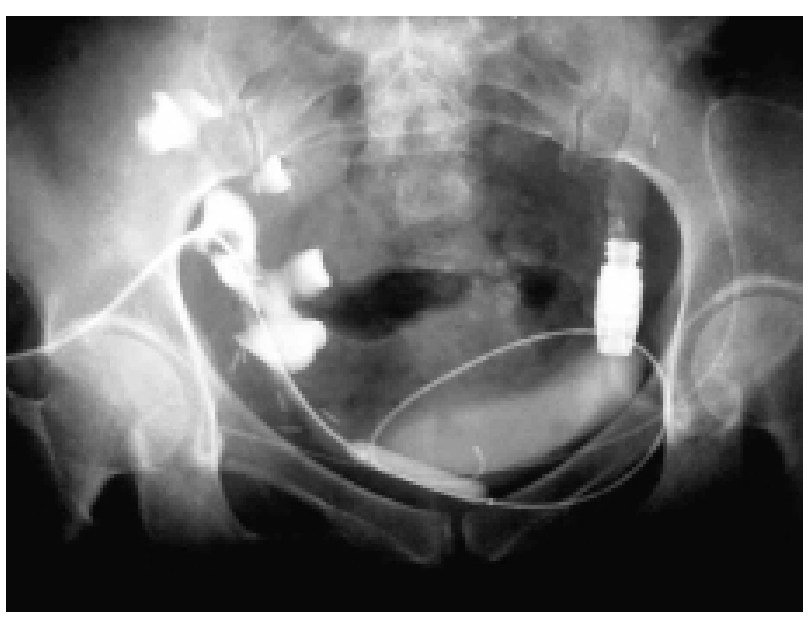

FIGURA 3. Dilatación con catéter-balón de alta presión la zona de estenosis ureterovesical.

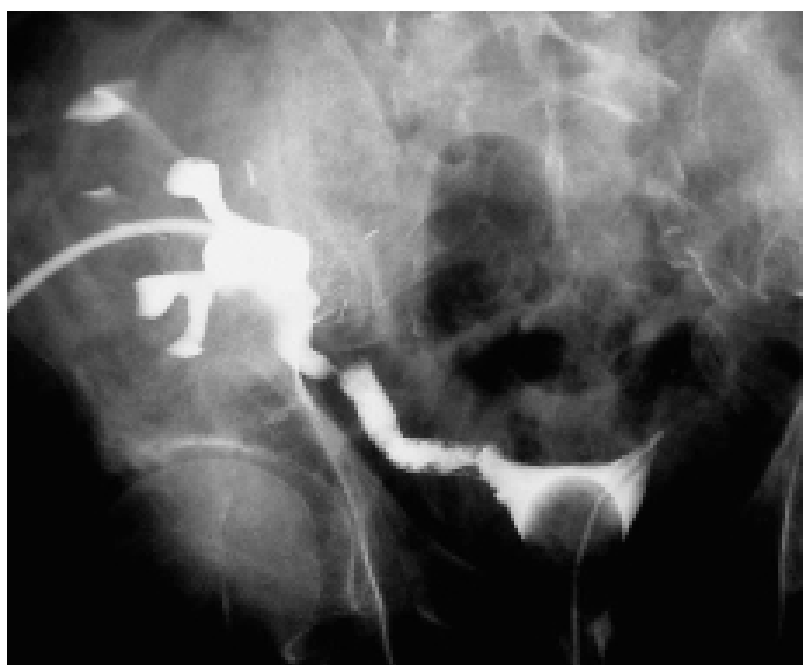

FIGURA 4. Pielografia de control postimplante. Se comprueba adecuado paso de contraste a vejiga confirmando permeabilidad de la prótesis.

que se resolvió con resección transuretral e implante del stent. El estudio histopatológico descartó recidiva tumoral.

A excepción de un caso, la estancia hospitalaria fue corta (entre 7 y 10 días). A los tres meses se realizó ecografía y analítica, sin evidencia de hidronefrosis ni deterioro de la función renal en ninguno de los pacientes.

Un paciente desarrolló en la evolución una estenosis ureteral proximal al stent, que precisó la colocación de catéter ureteral doble J, desestimándose implante de nueva prótesis por expectativa funcional renal corta, por lo que se mantuvo con cambios periódicos de catéter hasta vuelta a hemodiálisis a los 16 meses del implante. 
Otros dos pacientes volvieron a hemodiálisis por deterioro crónico del injerto a los 27 y 34 meses respectivamente, manteniendo permeable el stent en la última revisión.

El resto de pacientes mantienen función renal estable en la actualidad. En ningún caso se produjeron complicaciones importantes. Dos pacientes presentaron infección urinaria en el seguimiento sin repercusión funcional, resuelta con tratamiento antibiótico.

En ningún caso se presentó pérdida del injerto, ni deterioro funcional obstructivo secundario al implante del stent.

En la Tabla III se resumen los resultados y complicaciones.

\section{DISCUSIÓN}

El diagnóstico y tratamiento precoces de las estenosis ureterales post-TR son fundamentales para evitar la pérdida del injerto y disminuir la morbi-mortalidad asociada a este proceso ${ }^{3-5}$. Ante la elevación de productos nitrogenados, la ecografia, constituye el primer paso diagnóstico para establecer la existencia de obstrucción. El diagnóstico de estenosis ureteral se confirmará definitivamente con la realización de una pielografía anterógrada y colocación de nefrostomía que permite evaluar la recuperación funcional del injerto ${ }^{6,7}$.
Las opciones endourológicas para el tratamiento de las estenosis ureterales post-TR son varias: el implante de catéter ureteral tipo doble $\mathrm{J}$, la dilatación con un catéter balón de alta presión asociado catéter ureteral transitorio o el empleo de prótesis endoluminales autoexpandibles ${ }^{8,9}$.

Las endoprótesis (stent), se usan de rutina en cirugia vascular en el tratamiento de las oclusiones arteriales y venosas ${ }^{10} \mathrm{y}$ en la patología neoplásica de la vía biliar. En el tracto urinario, se han utilizado sobre todo para el tratamiento de la obstrucción uretral cervico-prostática con resultados satisfactorios en la mayoría de las series publicadas ${ }^{11}$.

Sin embargo, la experiencia existente con el uso de endoprótesis metálicas en el tracto urinario superior es limitado. La utilización de los mismos en el tratamiento de las estenosis ureterales postTR tiene un carácter anecdótico en la literatura.

El principal problema del empleo de prótesis metálicas a nivel ureteral, es la aparición en algunos casos de una hiperplasia urotelial que produce oclusión de la luz ureteral cubriendo el stent. Este proceso, en la mayoría de las ocasiones es transitorio pero a veces puede mantenerse en el tiempo.

Flueckinger et al. ${ }^{12}$ presenta una serie de 10 pacientes con obstrucción ureteral maligna tratados con endoprótesis metálicas. En 4 pacientes se observó a las dos semanas una oclusión de la luz

TABLA III

\begin{tabular}{|c|c|c|c|c|c|c|c|}
\hline & \multirow{2}{*}{$\begin{array}{l}\text { Tiempo desde } \\
\text { TR a implante } \\
\text { (Meses) }\end{array}$} & \multicolumn{2}{|c|}{$\begin{array}{l}\text { Creatinina } \\
(\mathrm{mg} / \mathrm{dl})\end{array}$} & \multirow{2}{*}{ Complicaciones } & \multirow{2}{*}{$\begin{array}{c}\text { Tiempo de } \\
\text { seguimiento }\end{array}$} & \multirow{2}{*}{$\begin{array}{l}\text { Vuelta a } \\
\text { diálisis }\end{array}$} & \multirow{2}{*}{ Permeabilidad } \\
\hline & & Pre & Post & & & & \\
\hline 1 & 52 (oct./95) & 6,5 & 4,1 & No & 27 & Si (enero 98) & $\mathrm{Si}$ \\
\hline 2 & 5 (oct./98) & 4,1 & 2,5 & No & 34 & Si (agosto 01) & $\mathrm{Si}$ \\
\hline 3 & 6 (marzo/99) & 5,7 & 2,3 & $\begin{array}{l}\text { Estenosis proximal } \\
\text { al stent a los } 4 \text { meses, } \\
\text { implante doble } \mathrm{J} \\
\text { Pielonefritis }\end{array}$ & 20 & $\begin{array}{c}\mathrm{Si} \\
\text { (nov./01) }\end{array}$ & $\mathrm{Si}$ \\
\hline 4 & 5 (mayo/00) & 2,6 & 1,41 & No & 22 & No & $\mathrm{Si}$ \\
\hline 5 & 72 (oct./01) & 3,75 & 1,6 & No & 8 & No & $\mathrm{Si}$ \\
\hline 6 & 73 (nov./01) & 4,23 & 1,8 & No & 9 & No & $\mathrm{Si}$ \\
\hline 7 & 18 (abril/02) & 5,7 & 1,8 & No & 1 & No & $\mathrm{Si}$ \\
\hline 8 & 26 (abril/02) & 2,8 & 1,6 & No & 1 & No & $\mathrm{Si}$ \\
\hline
\end{tabular}


ureteral a nivel del implante que precisó la derivación urinaria con catéter ureteral. Tras la retirada del mismo a los tres meses se comprobó radiológicamente el paso fluido de contraste a ese nivel.

En el resto de pacientes se produjo una reacción ureteral leve no obstructiva, observándose a los 6 meses que el stent se encontraba tapizado por una lámina de urotelio normal no obstructivo.

Para este autor, la extensión de la hiperplasia depende del grado de traumatismo urotelial provocado por el implante y el grado de distensión ureteral que se produce con el catéter de dilatación que se emplea en ocasiones previo a la colocación de la prótesis ${ }^{12}$.

Otro factor importante es que el stent debe quedar correctamente alineado con la pared ureteral, permitiendo un adecuado peristaltismo ureteral proximal y distal al uréter; si existen acodamientos aumenta el riesgo de reacción fibrótica y oclusión de la luz ureteral ${ }^{12}$.

En nuestra serie, el único paciente al que se le colocó el stent de menor calibre $(4 \mathrm{~mm})$ presentó una obstrucción post-implante que exigió la colocación transitoria de un catéter ureteral doble J, quedando totalmente permeable el stent tras la recuperación de la hiperplasia urotelial a las 6 semanas.

Otras posibles complicaciones derivadas son la reestenosis, la migración, las hemorragias y las infecciones ${ }^{13}$. En nuestra experiencia las complicaciones fueron menores, siendo las más frecuentes las infecciones sin repercusión sistémica ni deterioro funcional del injerto.

\section{CONCLUSIONES}

El tratamiento de las estenosis ureterales postTR mediante el empleo de stents, es una opción dentro de las posibilidades de manejo endourológico.

El empleo de prótesis metálicas autoexpandibles es un tratamiento seguro y efectivo, con baja morbi-mortalidad e indices bajos de complicaciones, siendo una alternativa razonable a otras opciones endourológicas, siempre que la selección del paciente sea la adecuada.

La reimplantación ureterovesical continúa constituyendo el tratamiento de elección de las estenosis ureterales post-TR en pacientes con función renal normal y sin comorbilidad asociada. Sin embargo en los pacientes con:
1. Deterioro crónico del injerto con expectativa funcional corta.

2. Pacientes con alto riesgo quirúrgico.

3 . Reestenosis tras reimplantación ureterovesical previa. El empleo de una prótesis metálica autoexpandible constituye la técnica de elección dada su eficacia y baja morbilidad asociada.

Si bien es cierto que son necesarios mayores periodos de seguimiento para establecer el resultado funcional a largo plazo.

\section{REFERENCIAS}

1. MAKISALO H, EKLUND B, SALMELA K et al.: Urological complications after 2084 consecutive kidney transplantation. Transplant Proc 1997; 29: 152-153.

2. SMITH RB, EHRLICH RM.: The surgical complications of renal transplantation. Urol Clin North Am 1979; 3: 621-646.

3. KASHI SH, LODGE JPA, GILES GR, IRVING HC.: Ureteric complications of renal transplantation. $\mathrm{Br} J$ Radiol 1992; 70: 139-143.

4. MUNDY AR, PODESTA ML, BEWICK M, RUDGE CJ, ELLIS FG.: The urological complications of 1000 renal transplants. Br J Radiol 1981; 53: 397-402.

5. HELLING TS, THOMAS CY, MOORE JD, KOONTZ PG.: The surgical approach to obstructive problems of the transplant ureter. Transplant Proc 1982; 14: 751-760.

6. SMITH TP, HUNTER DW, LETOURNEAU JG et al.: Urinary obstruction in renal transplant: diagnosis by anterógrada pyelography and results of percutaneous treatment. AJR 1988; 151: 507-510.

7. BOSMA RJ, VAN DRIEL MF, VAN SON WJ, DE RUITER AJ, MENSINK HJA.: Endourological management of ureteral obstruction after renal transplantation. $J$ Urol 1996; 15: 1099-1100.

8. OOSTERHHOF GO, HOITSMA AJ, WITJES JA et al.: Diagnosis and treatment of urological complications in kidney transplantation. Urol Int 1992; 49: 99.

9. BERENGUER A, LOVACO F, MAYAYO T et al.: La endourología en el trasplante renal. Arch Esp Urol 1986; Suppl. 2: 215.

10. WRIGHT KC.: Percutaneous transcatheter stent placement. Radiology 1990; 176: 620-621.

11. ASHKEN MH, COULANGE C, MILROY EJG, SARRAMON JP.: European experience with the urethral Wallstent for urethral strictures. Eur Urol 1991; 19: 181-185.

12. FLUECKINGER F, LAMMER J, KLEIN GE et al.: Malignant ureteral obstruction: preliminary results of treatment with metallic self-expandable stents. Radiology 1993: 173-186.

13. GORT HBW, MALI WPTM, VAN WAES PFGM. KLOET AG.: Metallic self-expandable stenting of a ureteroileal stricture. AJR 1990; 155: 422-423.

Dr. B. Pozo Mengual

C/ Camilo José Cela, 4 - Portal 2, 2으 B

28230 Las Rozas (Madrid)

(Trabajo recibido el 17 junio de 2002) 